\title{
Two Treatment Repeated Measurement Designs With Uncorrelated Observations: A Compact Review
}

\author{
Miltiadis S. Chalikias \\ Piraeus University of Applied Science \\ Email::mchalikias@hotmail.com, mchalik@teipir.gr
}

\begin{abstract}
Hedayat and Afsarinejad (1975,1978) introduced the model for repeated measurement designs without interactions which was the basis for the next researchers. We present the optimal designs for the direct and carry over effect of the model for 2,3, and 4 periods. Moreover, universally optimal designs, $\Phi$ optimal and balanced designs are also presented based on the conditions of Cheng and Wu (1980) and Chalikias and Kounias (2012).
\end{abstract}

Keywords: Repeated Measurements designs, direct effects, carry-over effects, universally optimality

\section{Introduction}

Repeated Measurements designs (or Cross-over designs or Change-over designs) are experimental designs where one of the $\mathrm{t}$ treatments is applied to one experimental unit for each of the $m \geq 2$ different periods.

If $\mathrm{n}$ is the number of experimental units, the design is denoted by RMD ( $t, n, m)$, where $\mathrm{t}$ is the number of treatments and $m$ the number of periods. Balaan (1965) is the first who considered the case of two periods in the model with treatment-period interaction. Some early papers in the field of design optimality from Kiefer and Wolfowitz (1960), Silvey and Titterington (1973) and Wynn (1977), Hedayat and Afsarinejad $(1975,1978)$ studied the case of universally optimal designs and balanced designs. Cheng and $\mathrm{Wu}(1980)$ examined universally optimal and strongly balanced designs. Carriere, K. C. \& Huang, R. (2000) considered two period designs for clinical trials Kunert (1983, 1984a, 1984b, 1991), Kunert, I. and Stufken. J. (2002), Laska and Meisner (1985), Kushner (1997a, 1997b, 1998), studied universally optimal designs. Mathews (1987, 1990), Kershner, and Federer, (1981) and Kushner (1997a) examined optimal designs of two treatments and gave optimal designs for 3 and 4 years in the case where the model errors are correlated. Finally, Azzalini and Giovagnoli (1987) and Williams and John (2007) are some of the researchers who dealt with the case of correlated errors.

The review papers of Stufken (1996) and Jones and Kenward (2014) as and the book of Diggle and Kenword 1994 give useful information and a plethora of reports on the subject.

\section{Definitions-Utility-Model}

In this design we are interested in the estimation of the direct effects, i.e. the effect of every treatment in the period which is applied, and the estimation of the carry over effects (or residual effect), i.e. the effect of every treatment in the period following the implementation period. Certainly a treatment may have an effect two periods later, but we will examine only the case of first order residual effects. The Repeated Measurements Designs (RMDs) have several advantages:

$>$ It takes only experimental $n$ (experimental units) for nxm observations

$>$ If the variability within each experimental unit is less than the variability between experimental units, then RMDs give accurate estimates of some variables.

$>$ RMDs are used in clinical trials or in the pharmaceutical industry, where the purpose is the improvement of chronic diseases and not the cure.

The disadvantages are:

$>$ The duration of the experiments is long, so the danger of withdrawals is significant 
Over time the conditions change. So the values of some variables must also change. This is partially corrected by entering in the model treatment-period interaction. The model without interactions, introduced by Hedayat and Afsarinejad $(1975,1978)$, is:

$$
Y_{i j k}=\mu+\tau_{i j}+\pi_{j}+\delta_{i, j-1}+\gamma_{i k}+e_{i j k}
$$

where i refers to the number of the treatment sequence $i=0,1, \ldots, 2^{m}-1$

$j$ refers to the number of periods, $\mathrm{j}=1,2, \ldots \mathrm{m}$

$k$ refers to the number of the experimental units, $\mathrm{k}=1,2, \ldots \mathrm{n}$

$\mu$ is the mean,

$\tau_{i j}$ is the effect of the $\mathrm{i}$-th treatment applied on the $\mathrm{j}$-th period,

$\delta_{i, j-1}$ is the carry-over effect of the i-th treatment applied in the previous period, i.e. the period $\mathrm{j}-1$.

$\pi_{j}$ is the effect of the $\mathrm{j}$ - period

$\gamma_{i k}$ is the effect the of the i-th sequence when it is applied on the k-th experimental unit, and $e_{i j k}$ is the error.

In the model independent observations within each experimental unit are considered. If the $\mathrm{k}$ experimental unit is randomly selected, then the impact of facility $\zeta_{k}$ (a random variable) must also be considered. The model becomes:

$$
Y_{i j k}=\mu+\tau_{i j}+\pi_{j}+\delta_{i, j-1}+\gamma_{i}+\zeta_{k}+e_{i j k}
$$

Remarks:

> Bose, M. and Mukherjee, B (2000) examined the estimation of higher order carry-over effects (of course the model was different).

$>$ If the $\gamma_{i k}$ effect is constant, all experimental units used in each sequence will have the same effect, called the impact of sequence. Regarding the optimal design, this is not a limitation, because it is shown (Kounias and Chalikias 2014) that in both cases the optimal design is the same.

$>$ In order to avoid carry-over effects, an interim period is used, after the end of each of the first m1 periods. This is the washout period (wash out or rest period) which renders the carry-over effect in the next period negligible.

When there are two treatments, two periods, and the model has treatment-period interaction, then the carry-over effects can't be estimable. Freeman (1989) suggested the existence of an additional observation before the first period. These observations obtained before the first period and at the end of each period are called baseline measurements. For more about Baseline measurements see Liang, Y. and Carriere, K. C. (2010).

$>$ In the case of two treatments, Fleis (1989) remarked that when we consider the two sequences $\mathrm{AA}$, and $\mathrm{AB}$, then the carry-over effect of $\mathrm{A}$ is not necessary the same in both cases.

$>$ There is no carry-over effect in the first period and this leads some researchers to assume arbitrarily the condition $\delta_{\mathrm{A}}+\delta_{\mathrm{B}}=0$ for carry-over effects (Mathews 1987, 1990, Jones and Kenward, 2003, p.119, 121). This is avoided in the cyclic experiments repeated measurements, where the first period becomes the last of the cycle (Magda, 1980). Another way is to have a pre-period, (Kunert 1983, Sinha 1975), in which the treatment applied is the same as in the last period.

For review papers see Mathews (1994), Stufken (1996).

\section{$3 \quad$ Brief History of the Method}

Although these designs are used more in clinical trials, the first known application was in agriculture by Lawes and Gilbert (1864) in England. Lawes and Gilbert divided in two sections a field. To the first section they applied ammonia in the first year and potash in the next year, while to the other section they applied potash in the first year and ammonia in the next year. They noticed that the section where 
they had applied the potash-ammonia treatment had yielded more than the section where the ammoniapotash treatment was applied.

Simpson (1938) used four diets on 24 children. There are 24 permutations and each child took, in four successive periods, one of the 4-diets (treatments) permutations. Measurements were made at the end of each period. Simpson was the first who mentioned the concept of the carry-over effects. To eliminate it, he proposed the implementation of the washout periods.

Cochran, Autrey and Cannon (1941) used cows as experimental units and compared three diets A, B, $\mathrm{C}$, in a three-period experiment. In their design, they used 2 orthogonal 3x3 Latin squares, as given in the following Table 1. The design is uniform and balanced, i.e. in each period each treatment appears twice and each treatment is applied once to each experimental unit (uniform design). Also each of the 6 pairs AB, BA, AC, CA, BC, CB appears twice across 6 experimental units (balanced design).

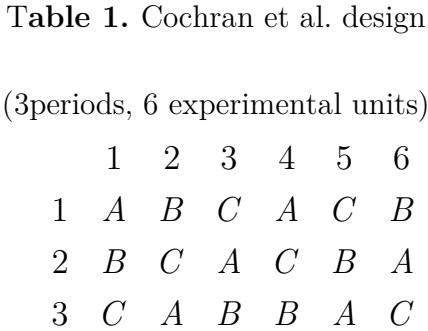

Uniform and balanced designs were used extensively by researchers (see section 4). This property is used to give the same accuracy in the estimation of the differences of direct effects. However, as we shall see, uniform and balanced designs do not always offer the lowest variance for the estimated parameter.

Cochran et al (1941) were the first who introduced the carry-over effects in the model and showed that for the RMDs $(\mathrm{t}, \mathrm{n}=\mathrm{t}(\mathrm{t}-1), \mathrm{m}=\mathrm{t})$, there exist $\mathrm{t}-1$ Latin squares which correspond to uniform and balanced designs.

Williams (1949) showed that if the number of treatments is even and $t=m$, balanced designs can constructed from one Latin square. When the number of treatments is odd, then at least two Latin must be used squares for the construction of balanced designs.

Williams (1950) found designs for estimating second-order carry-over effects, and he showed how balanced designs using the minimum number of experimental units can be constructed. Quenouille (1953) produced designs for $\mathrm{m}=2,3$, and 4 (for further details see John and Quenouille, second edition, 1977, first edition, 1953). A list of balanced designs is given by Patterson and Lucas $(1959,1962)$, the list is contained in the book of Ratkowsky, Evans and Alldredge (1993).

Table 2. The design of Quenouille for $t=2$

\begin{tabular}{|c|c|c|c|}
\hline & 1 & 2 & 3 \\
\hline 1 & $A$ & $B$ & $B$ \\
\hline 2 & $A$ & $A$ & $B$ \\
\hline 3 & $B$ & $A$ & $A$ \\
\hline 4 & $B$ & $B$ & $A$ \\
\hline
\end{tabular}

Patterson and Lucas (1959) gave strongly balanced designs for the estimation, with the same precision, of both direct effects and the first-order carry-over effects. In these designs each treatment is followed by every other treatment an equal number of times, and the treatment of the last period is the same as the treatment of the penultimate period, (see table 3). In the design of Table 3 each treatment is followed from all other treatment 2 times. 
Table 3. The design of Patterson and Lucas

$\begin{array}{ccccccc}\text { Experimental units (4 periods) } \\ & 1 & 2 & 3 & 4 & 5 & 6 \\ 1 & A & B & C & A & C & B \\ 2 & B & C & A & C & B & A \\ 3 & C & A & B & B & A & C \\ 4 & C & A & B & B & A & C\end{array}$

Balaan (1968), in order to study the interaction of the direct effect and the period, proposed designs for two periods where the number of experimental units is $t^{2}$, i.e. $\operatorname{RMD}\left(t, t^{2}, 2\right)$. A Balaan design contains all $t^{2}$ sequences of $\mathrm{t}$ treatments, the design for $\mathrm{t}=2$ is given in Table 4 .

Table 4. The design of Balaan

Experimental units (2 periods)

$\begin{array}{rllll} & 1 & 2 & 3 & 4 \\ 1 & A & B & A & B \\ 2 & A & B & B & A\end{array}$

\section{Optimal Designs}

A significant change in the direction of research in repeated measurements designs happened when Hedayat and Afsarinejad $(1975,1978)$ applied the theory of optimal design problems of RMD (t, n, m).

If $\theta$ is all or some of the parameters of a linear model with errors following the multidimensional normal distribution $N\left(0, \delta^{2} \Sigma\right)$, where $\Sigma$ is known, then, the generalized least squares estimator $\hat{\theta}$ can be find. The covariance matrix of $\hat{\theta}$ is $\operatorname{var}(\hat{\theta})=\sigma^{2} \mathbf{C}$ where $\mathbf{C}$ is the information matrix (Shah and Sinha, 1989).

Then the design which minimizes $\varphi(\mathbf{C})$ for every continuous decreasing function $\varphi$ is called $\Phi$ optimal design.

Some well known optimization criteria are:

D-optimization, where $\varphi(\mathbf{C})=\operatorname{det} \mathrm{C}$,

A-optimization when $\varphi(\mathbf{C})=\operatorname{tr} \mathrm{C}$,

E-optimization, where $\varphi(\mathbf{C})=$ maximum eigenvalue of $\mathrm{C}$.

Kiefer (1975) gave the criteria for a universally optimal design $\mathrm{d}^{*}$, ie $\varphi\left(\mathbf{C}_{d^{*}}\right) \leq \varphi\left(\mathbf{C}_{d}\right)$ for all these convex functions $\varphi$. So $\mathrm{d}^{*}$ is optimal according to the three criteria D, A, E.

The universally optimal designs are usually uniform and balanced in accordance with the following definitions (Chalikias and Kounias 2008b, 2017):

An RMD (t, n, m) is uniform, on the periods when in each period each treatment applies to the same number of experimental units.

An RMD ( $t, n, m)$ is uniform on the units when to every experimental unit each treatment is applied the same number of periods.

An RMD ( $\mathrm{t}, \mathrm{n}, \mathrm{m})$ is uniform when it is uniform on the periods and uniform on the experimental units. Tables 1 and 2 give uniform designs, while tables 3 and 4 give uniform designs only on the periods.

A design $d$ with a kxk information matrix $C_{d}$ and eigenvalues $\lambda_{1}, \lambda_{2}, \ldots \lambda_{\kappa}$ is $\Phi$-optimal in the class of F designs, if it minimizes $\sum_{i=1}^{\kappa} \varphi\left(\lambda_{i}\right)$ for all continuous decreasing convex functions $\varphi(\lambda)$ in the class of F. $\Phi$ optimality is a weaker condition than universal optimality. For example MV-optimality is covered by universal optimality but not by $\Phi$-optimality (Chalikias and Kounias 2012) 
Also in many papers appears an extensive use of balanced and strongly balanced (completely balanced) designs. An RMD (t, n, m) is called balanced if the number that an arranged pair of treatments (different treatments) is applied is the same for all such pairs. In order for a design RMD (t, n, m) to be balanced, the condition $\lambda \cdot t \cdot(t-1)=n \cdot(m-1)$ must hold for some value $\lambda$.

An RMD ( $t, n, m)$ is called strongly balanced if the number that an arranged pair of treatments (different or same treatments),) is applied is the same for all pairs. The design in table 1 is balanced and each pair $\mathrm{AB}, \mathrm{BA}, \mathrm{AC}, \mathrm{CA}, \mathrm{BC}, \mathrm{CB}$ appears 2 times. The designs in tables 2,3 , and 4 are strongly balanced.

Hedayat and Afsarinejad (1978) consider independent errors with constant dispersion and show that some balanced designs in the class of uniform designs RMD $(t, n=\lambda t, m=t)$, are universally optimal : a) To estimate the direct effects, b) to estimate the carry over effects. They also give examples of universally optimal designs $\operatorname{RMD}(\mathrm{t}, \mathrm{t}, \mathrm{t})$ and $\operatorname{RMD}(\mathrm{t}=2 \mathrm{t}+1, \mathrm{n}=2 \mathrm{t}, \mathrm{m}=\mathrm{t})$.

Cheng and $\mathrm{Wu}(1980)$ extend the results of Hedayat and Afsarinejad and show that strongly balanced, uniform designs $\operatorname{RMD}(\mathrm{t}, \mathrm{n}, \mathrm{m})$ are universally optimal for estimation of the direct and carry-over effects. Chalikias and Kounias (2012) extend the conditions of Cheng and $\mathrm{Wu}$ in order to include the case of $\mathrm{n}$ even and $n=2 \bmod 4$. Moreover they show that these conditions are necessary to construct $\Phi$-optimal designs.

Kunert (1983,1984,1985), Hedayat and Zhao (1990), Stufken (1991), Carriere and Reinsel (1992, 1993), Kushner (1997a, 1997b, 1998, 1999), Kunert and Stufken (2002 ), Hedayat and Yang (2003, 2004), deal with finding optimal RMD ( $\mathrm{t}, \mathrm{n}, \mathrm{m})$ designs, or construct universally optimal designs from the class of uniform designs.

Almost all researchers of $\operatorname{RMD}(\mathrm{t}, \mathrm{n}, \mathrm{m})$ designs, as mentioned above, are studying universally optimal designs using the approximate theory designs and applying the theorem of Kiefer (1975) to uniform designs, thus limiting the class of RMD ( t , n, m). For instance, Hedayat and colleagues $(1975,1978$, 1990，2003，2004) study the class RMD (t, n, $\mathrm{m}=\mathrm{t}$ ). The universally optimal designs given refer to specific values of the parameters $t, n, m$ and when they exist they are applied to a limited class of discrete designs and not for all values of $\mathrm{t}, \mathrm{n}, \mathrm{m}$.

\section{The Case of Two Treatments}

For two periods there are 4 treatment sequences, AA, AB, BA, BB. Generally, for $m$ periods, there exist $2^{m}$ sequences. In order to have a stable and easy way of counting sequences, Kounias and Chalikias (2008a) used the binary numbering system, putting 0 for $\mathrm{A}$ and 1 for B. Thus we have the matches $A A \leftrightarrow 0, B A \leftrightarrow 1 A B \leftrightarrow 2, B B \leftrightarrow 3$ for example for a 5 period sequence BABBA corresponds to the number 13 because

$$
B A B B A \leftrightarrow 1 \cdot 2^{0}+0 \cdot 2^{1}+1 \cdot 2^{2}+1 \cdot 2^{3}+0 \cdot 2^{4}=13
$$

With that enumeration, the numbers $i, 2^{m}-1-i, i=0,1, \ldots, 2^{m-1}-1$ where $\mathrm{m}$ is the number of periods correspond to pairs of dual sequences.

\section{Two Periods}

The $2 \times 2$ designs, i.e. RMD $(t=2, n, m=2)$, have been studied by many researchers (see Jones and Kenward, 2003, chapter 2, which examines the balanced design AB, BA). Kounias and Chalikias (2008) show that for the model without interactions of direct effects-period, the optimal designs for estimating the direct effects are:

a) For $n=$ even: On $k$ experimental units the sequences $A A$, and $A B$ are applied and on $(n-2 k) / 2$ experimental units the sequences $\mathrm{BA}, \mathrm{BB}$, are applied. The dispersion is

$$
\operatorname{var}\left(\hat{\tau}_{\mathrm{A}}-\hat{\tau}_{\mathrm{B}}\right)=\sigma^{2} 8 / n
$$

b) For $\mathrm{n}=$ odd: On (n-1)/2 experimental units the sequences $\mathrm{AA}$, and $\mathrm{AB}$ are applied and on $(n+1) / 2$ experimental units the sequences $\mathrm{BA}, \mathrm{BB}$ are applied. The dispersion is

$$
\operatorname{var}\left(\hat{\tau}_{\mathrm{A}}-\hat{\tau}_{\mathrm{B}}\right)=\sigma^{2} 8 /\left(n-\frac{1}{n}\right)
$$


If there is interaction in the model, then only the difference $\left(\tau_{\mathrm{A}}-\tau_{\mathrm{B}}\right)-(\tau \pi)_{A 1}$ is estimable and it has the previous optimal designs with the same dispersion.

For carry-over effects $\delta_{\mathrm{A}}, \delta_{\mathrm{B}}$, it is shown that only $\delta_{\mathrm{A}}-\delta_{\mathrm{B}}$ is estimable in the model without interaction, with optimal design:

c) For $\mathrm{n}=$ even: On $\mathrm{n} / 2$ experimental units the sequences $\mathrm{AA}, \mathrm{BB}$ are applied. The dispersion is $\operatorname{var}\left(\hat{\delta}_{\mathrm{A}}-\hat{\delta}_{\mathrm{B}}\right)=\sigma^{2} 8 / n$

d) For $n=$ odd: On (n-1)/2 experimental units the sequence AA is applied and on $(n+1) / 2$ experimental units the sequence BB is applied. The dispersion is $\operatorname{var}\left(\hat{\tau}_{\mathrm{A}}-\hat{\tau}_{\mathrm{B}}\right)=\sigma^{2} 8 /\left(n-\frac{1}{n}\right)$.

In the model with interaction, $(\tau \pi)_{A 1}$ indicates that only the difference $\delta_{\mathrm{A}}-\delta_{\mathrm{B}}-2(\tau \pi)_{\mathrm{A} 1}$ is estimable with optimal designs and dispersions as in (c), (d). It is shown that these designs are not balanced, except (a) for $\mathrm{k}=\mathrm{n} / 2$. Moreover, the optimal designs for direct effects are different from the designs for the carry-over effects. Designs, in (a) only for $\mathrm{n}=0 \bmod 4$, and (c) only for $\mathrm{n}$ even, are given by Laska, Meisner and Kushner (1983) and Kunert (1984). Balaan (1965, 1968), Cheng and Wu (1980), Laska, Meisner and Kushner (1983) give as optimal for the estimation of both parameters $\left(\tau_{\mathrm{A}},\left(\delta_{\mathrm{A}}-\delta_{\mathrm{B}}\right)\right)$, the strongly balanced design with $\mathrm{n} / 4$ experimental units in each of the sequences $\mathrm{AA}$, $\mathrm{AB}, \mathrm{BA}, \mathrm{BB}$, i.e. only for $\mathrm{n}=0 \bmod 4$. Furthermore, JF Reed (2004) studied the effects of wash out period for the case of two treatments while Candel, MJJM (2012), Lalou 2012 and Bardzokas \& Lalou $(2007,2009)$ dealt with the application of the design of Balaam in mixed models.

For every value of $\mathrm{n}$, it is shown that the numbers of experimental units corresponding to the sequences $\mathrm{AA}, \mathrm{AB}, \mathrm{BA}, \mathrm{BB}$ in the D-optimal design for the estimation of both parameters $\left(\tau_{\mathrm{A}},\left(\delta_{\mathrm{A}}-\delta_{\mathrm{B}}\right)\right)$ are equal or differ one experimental unit.

\section{$7 \quad$ Three Periods}

In the case of three periods, $\operatorname{RMD}(2, \mathrm{n}, 3)$, the carry-over effects $\delta_{\mathrm{A}}, \delta_{\mathrm{B}}$ are not estimable, while the difference $\delta_{\mathrm{A}}-\delta_{\mathrm{B}}$ is estimable.The optimal design, in the model without interaction, for both the direct effect $\left(\tau_{\mathrm{A}}-\tau_{\mathrm{B}}\right)$ and $\left(\delta_{\mathrm{A}}-\delta_{\mathrm{B}}\right)$, (Chalikias and Kounias 2017), are:

$\alpha$ ) For $n=$ even: On $n / 2$ experimental units the sequences ABB, BAA are applied. The variance is $\operatorname{var}\left(\hat{\tau}_{\mathrm{A}}-\hat{\tau}_{\mathrm{B}}\right)=\sigma^{2} 3 /(2 n), \operatorname{var}\left(\hat{\delta}_{\mathrm{A}}-\hat{\delta}_{\mathrm{B}}\right)=\sigma^{2} 2 / n$ correspondingly.

$\beta)$ For $n=$ odd: On $(n+1) / 2$ experimental units the sequence ABB is applied and on $(n-1) / 2$ experimental units the sequence BAA is applied.

The variance is $\operatorname{var}\left(\hat{\tau}_{\mathrm{A}}-\hat{\tau}_{\mathrm{B}}\right)=\sigma^{2} 3 /(2(n-1 / n)), \operatorname{var}\left(\hat{\delta}_{\mathrm{A}}-\hat{\delta}_{\mathrm{B}}\right)=\sigma^{2} 2 /(n-1 / n)$ correspondingly.

Kershner and Federer (1981), dealt with 6 designs with names D2.3.3, D 2.3.4, D2.3.5, D4.3.1, D4.3.2, D4.3.3, D6.3.1. From these, D 2.3.4 is optimal, while the other 5 are not optimal. D2.3.4 was given by Lucas (1957). Also, the optimal design BAA, ABB, for $n$ even is given by Laska et al. (1983), Laska and Meisner (1985), Mathews (1987, 1990), and Kushner (1997a). This design is universally optimal, as shown by Cheng and Wu (1980, Corollary 3.3.1).

Chalikias and Kounias 2012 extended the conditions of Cheng and $\mathrm{Wu}$, in order to present a method to construct $\Phi$-optimal designs for the case of $\mathrm{p}=3$ and $\mathrm{n}=0 \bmod 4$. Moreover Kounias and Chalikias 2015 extract the universally optimal designs for the model with interactions.

\section{Four Periods}

For the model without interaction, Quenouille (1953) gives the design ABBA BAAB, AABB, BBAA for $\mathrm{n}=0 \bmod 4$ with $\mathrm{n} / 4$ experimental units in each sequence. This design satisfies the relations of Cheng and $\mathrm{Wu}$ because $u_{3}=u_{12}=n / 4, u_{5}=u_{10}=0, u_{6}=u_{9}=n / 4$. This solution was also given by Laska, Meisner and Kushner (1983), Mathews (1990), Laska and Meisner (1985). 
The theorem of Laska and Meisner (1985) gives universally optimal designs when the number n of experimental units is even, but not for all cases, as is the case with $\mathrm{m}=4$ shows. Finally, Chalikias and Kounias (2012) construct $\Phi$-optimal designs for the case of $m=4$ and $n=2 \bmod 4$.

\section{Other Topics of Interest}

Other topic of interest is of course the extension of the process for five numbers of periods. Moreover the process can be extended for slight different models. Particularly model with two treatments and correlated observations, model with two treatments with interaction and of course the extension of these for three treatments.

\section{References}

1. Azzalini, A. and Giovagnoli, A. (1987). Some optimal designs for repeated measures with autoregressive errors. Biometrika, 74, 725-734.

2. Balaan, L.N. (1965). A Two-Period Design for Treatment by Period Interaction. Paper No. BU-177-M, Biometrics unit, Kent University.

3. Balaan, L. N.(1968). A two period design with $t^{2}$ experimental units. Biometrics unit, Cornell University 24, 61-73.

4. Bardzokas D., Lalou P. (2009) The method of Complex Analysis for the solution of plane problems of the theory of Thermoconductivity and Thermoelasticity for multiply connected bodies, Proceeding of National Academy of sciences of Armenia, Mechanics, No4. Vol 62, 23-41.

5. Bardzokas D., Lalou P. (2007) The influence of coupled thermal fields on the behavior of cracked bodies, Proceedings of $4^{\text {th }}$ National conference on NDM

6. Bose, M.and Mukherjee, B(2000). Cross over designs in the presence of higher order carey overs. ANZ Journal of Statistics. 42(1): 235-244

7. Candel, M.J.J.M. 2012 Parallel, AA/BB, AB/BA and Balaam's design: Efficient and maximin choices when testing the treatment effect in a mixed effects linear regression Pharmaceutical Statistics Volume 11, Issue 2, pages 97-106, March/April 2012.

8. Carriere C., Huang R. (2000) Crossover designs for two treatment clinical trials. Journal of Statistical planning and inference. 87 (1): 125134.

9. Carriere K. C. and Reinsel G. C. (1992). Investigation of dual-balanced crossover designs for two treatments. Biometrics 48, 1157-1164.

10. Carriere K. C. and Reinsel G. C. (1993). Optimal two period repeated measurement designs with two or more treatments. Biometrika, 80, 4, 924-929.

11. Chalikias M., and Kounias S.(2012). Extension and necessity of Cheng and Wu conditions Journal of Statistical Planning and Inference 142 (7), pp. 1794-1800.

12. Chalikias M., Kounias S., (2017). Optimal two Treatment Repeated Measurement Designs for three Periods Communications in Statistics - Theory and Methods Vol 46, Pages: 200-209

13. Cheng, C.S. and Wu C.F. (1980 ). Balanced repeated measurements designs. Ann. Statist 11, 29-50. Correction (1983) $11,349$.

14. Cochran, W.G., Autrey, K.M., and Cannon, C.Y. (1941). A double change-over design for dairy cattle feeding experiments. J. Dairy Sci., 24, 937-51.

15. Diggle, P., \& Kenward, M. G. (1994). Informative drop-out in longitudinal data analysis. Applied statistics, 4993.

16. Fleis, J.L. (1989). A critique of recent research on the two-treatment crossover design Controlled Clinical Trials 10, 237-243.

17. Freeman, P.R. (1989). The performance of the two-stage analysis of two-treatment, two-period crossover trials. Statistics in Medicine 8, 1421-1432.

18. Hedayat, A. S.and Afsarinejad K.(1975). Repeated measurements designs I. A survey of Statistical Designs and Linear Models, J.N. Srivastava editor, North-Holland, Amsterdam, 229-242.

19. Hedayat, A. S. and Afsarinejad K.(1978). Repeated measurements designs II. Ann. Statist. 18, 1805-1816. 
20. Hedayat, A. and Zhao W. (1990). Optimal two period repeated measurements designs. Ann. Statist. vol. 18, No. 4, 1805-1816.

21. Hedayat, A. S. and Yang, M. (2003).Universal Optimality of Balanced Uniform Crossover Designs. Ann. Statist., 31, 978-983.

22. Hedayat, A. S. and Yang, M. (2004).Universal Optimality of Selected Crossover Designs. J. Am. Statist. Assoc., vol. 99, No 466, 461-466.

23. John, J.A., Quenuille, M.H. (1977). Experiments: Design and Analysis. Charles Griffin \& Co. Ltd, London.

24. Jones, B., \& Kenward, M. G. (2014). Design and analysis of cross-over trials. CRC Press.

25. Kiefer, J. (1975). Construction and optimality of generalized Youden squares. A survey of Statistical Design and Linear Models. J.N. Srivastava, Editor, North-Holland, Amsterdam, 333-353.

26. Kershner, R.P. and Federer, W.T. (1981). Two-treatment Crossover Design for Estimating a variety of Effects. J. Am. Statist. Assoc., vol 76, 612-619.

27. Kiefer, J., \& Wolfowitz, J. (1960). The equivalence of two extremum problems. Canadian Journal of Mathematics, 12(5), 363-365.

28. Kounias, S \& Chalikias, M (2008a). Optimal and universally optimal two treatment repeated measurement designs. F. Vonta et al., editors, Statistics for industry and technology Birkhauser, Boston, Basel, Berlin, 465-477.

29. Kounias, S., Chalikias M., (2008b), Estimability of parameters in a linear model and related characterisations, Statistics and Probability Letters 78 (15):2437-2439

30. Kounias, S., \& Chalikias M.S.,(2015) "Optimal two treatment repeated measurement designs with treatmentperiod interaction in the model" Utilitas Mathematica, 96, 243-261.

31. Kunert, J. (1983). Optimal design and refinement of the linear model with applications to repeated measurements designs. Ann. Statist., 11, 247-257.

32. Kunert, J. (1984a). Optimality of balanced uniform repeated measurements designs. Ann. Statist., 12, 1006-1017.

33. Kunert, J. (1984b). Optimal repeated measurements designs for correlated observations and analysis by weighted least squares. Biometrika 72, 375-389.

34. Kunert, J. (1991). Cross-over designs for two treatments and correlated errors. Biometrika 78, 315-324.

35. Kunert I and Stufcken, J (2000). Optimal cross over designs in a model with self and mixed carry over effects. Journal of the American Statistical Association 459 (97): 898-906.

36. Kushner, H.B. (1997a). Optimality and Efficiency of the Two Treatment Repeated Measurements Design. Biometrika, 84, 455-468.

37. Kushner, H.B. (1997b). Optimal Repeated Measurements Designs: The Linear Optimality Equations.. Ann. Statist., 25, 2328-2344.

38. Kushner, H.B. (1998). Optimal and Efficient Repeated- Measurements Designs for Uncorrelated observations. J. Am. Statist. Assoc, Vol. 93, 443, 1176-1187.

39. Kushner, H.B. (1999). H-symmetric optimal repeated measurements designs Journal of statistical planning and inference, Vol. 76, issues1-2 pages 235-261.

40. Lalou P., (2012) Integral equations of the problem thermoelasticity in cracked isotropic plate with inclusion, Applied Mathematical Sciences, Vol 6, No 62, 3081-3093.

41. Laska, E.M., Meisner M. and Kushner H.B. (1983). Optimal crossover designs in the presence of carryover effects. Biometrics 39, 1087-1091.

42. Laska, E.M. and Meisner M. (1985). A variational approach to optimal two treatment cross-over designs: application to carryover effect models. J. Am. Statist. Assoc. 80, 704-710.

43. Lawes, J.B. and Gilbert, J.H. (1864). Report of experiments on the growth of wheat for twenty years in succession on the same land. Journal of theAgricultural Society of England, 25, 93-185, 449-501.

44. Liang, Y. and Carriere, K. C. (2010), On the Role of Baseline Measurements for Crossover Designs under the Self and Mixed Carryover Effects Model. Biometrics, 66: 140-148.

45. Liebing, J. (1847). Chemistry in its Application to Agriculture and Physiology, 4th edition, Taylor and Walton, London.

46. Longford N. (1993). Random effects models. Oxford Science Publications.

47. Lucas, E.L. (1957). Extra-Period Latin-Square Change over design. Journal of Dairy Science, 40, 225-239.

48. Magda, C.G. (1980). Circular balanced repeated measurements designs. Commun. Statist.-Theor. Meth. A9, 1901-1918. 
49. Mathews, J.N.S. (1987). Optimal crossover designs for the comparison of two treatments in the presence of carryover effects and autocorrelated errors. Biometrika, 74, 2, 311-320.

50. Mathews, J.N.S. (1990). Optimal dual-balanced two treatment crossover designs. Sankhya, 52, B, 3, 332-337.

51. Mathews, J.N.S. (1994). Modeling and optimality in the design of crossover studies for medical applications. Journal of Statistical Planning and Inference, 42, 89-108.

52. Patterson, H. D. and Lucas, H. L.(1959). Extra period change-over designs. Biometrics, 15, 116-132.

53. Patterson, H. D. and Lucas, H. L.(1962). Change-over designs. North Carolina Agricultural Experimental Station, Tech. Bull. No 147.

54. Ratkowsky, D.A., Evans, M.A., Alldredge, J.R. (1999). Cross-Over Experiments. Marcel Dekker, Inc.

55. Reed J.F(2004)Analysis of two-treatment, two-period crossover trials in emergency medicine Annals of Emergency Medicine Volume 43, Issue 1, January, Pages 54-58

56. Queuneille h. (1953) The design analysis of experiments. London: Charles Griffin and Company Ltd

57. Simpson., T.W. (1938). Experimental methods and human nutrition (with discussion). Journal of the Royal Statistical Society, Series B, 5, 46-69.

58. Shah, K.R. and Sinha, B.K. (1989). Theory of Optimal Designs. Lecture Notes in Statistics. Springer, New York.

59. Sinha, B.K. (1975). On some optimum properties of serially balanced designs. Sankhya Ser. B, 37, 173-192.

60. Silvey, S. D., \& Titterington, D. M. (1973). A geometric approach to optimal design theory. Biometrika, 60(1), 21-32.

61. Stufken, J.(1991). Some families of optimal and efficient repeated measurements designs. Journal of Statistical Planning and Inference, 27, 75-83.

62. Stufken, J.(1996). Optimal crossover designs. Design and Analysis of Experiments. Handbook of Statistics 13, (S. Ghosh and C.R.Rao, eds.), 63-90, North-Holland, Amsterdam.

63. Williams, E.J.(1949). Experimental designs balanced for the estimation of residual effects of treatments. Aust. J. Sci. Res., A, 2, 149-168.

64. Williams, E.J.(1950). Experimental designs balanced for pairs of residual effects. Aust. J. Sci. Res., A, 3, 351363.

65. Williams, E.R. and John, J.A. (2007), Construction of crossover designs with correlated errors Australian \& New Zealand Journal of Statistics, 49: 61-68.

66. Wynn, H. P. (1977). Minimax purposive survey sampling design. Journal of the American Statistical Association, $72(359), 655-657$ 